\title{
Early Results of the Attune Knee System: A Minimum 2 Year Follow Up Observational Study
}

\author{
James Sires ${ }^{1 *}$, Stephanie Lennon ${ }^{2}$, Mark Inglis ${ }^{3}$, Anh-Minh Nguyen ${ }^{4}$ and Christopher Wilson ${ }^{5}$ \\ ${ }^{1}$ Medical Student, College of Medicine and Public Health, Flinders University, Australia \\ ${ }^{2}$ Orthopaedic Registrar, Department of Orthopaedics, Repatriation General Hospital, Australia \\ ${ }^{3}$ Orthopaedic Consultant, Department of Orthopaedics, Repatriation General Hospital, Australia \\ ${ }^{4}$ Statistician, College of Medicine and Public Health, Flinders University, Australia \\ ${ }^{5}$ Orthopaedic Consultant, Department of Orthopaedics, Repatriation General Hospital, Australia and College of Medicine and \\ Public Health, Flinders University, Australia
}

\begin{abstract}
Background: The ATTUNE Knee System was designed to improve patient outcome and satisfaction. The aims of this study were to assess patient outcome after receiving an ATTUNE total knee replacement (TKR) and ensure early results were comparable to other TKR systems in Australia.

Methods: 332 ATTUNE TKR's were performed locally, mean follow-up was 2.6 years (2.0 to 3.2). Revision data was collected on all ATTUNE TKR's. ATTUNE TKR's performed at our university teaching hospital $(n=162)$, had patient reported outcome measured using the Multi-Attribute Arthritis Prioritisation Tool (MAPT) questionnaire.
\end{abstract}

Results: Revision rate of the ATTUNE TKR was similar to national rates (1.6\% vs. $2.1 \%)(p=0.508)$. Postoperative MAPT scores were significantly lower after TKR ( $n=87)$ (median 63.4 vs. 0.0$)(p<0.001)$. A total of $82(94.3 \%)$ people had an improved MAPT score post-TKR.

Conclusion: Our findings suggest the ATTUNE TKR has comparable revision rates to other TKRs currently available in Australia. Furthermore, patient reported outcome was good 2.4 years postoperatively.

KEYWORDS: Total knee arthroplasty; ATTUNE Knee System; Revision rates; Patient reported outcomes; Multi-attribute arthritis prioritisation tool

\section{INTRODUCTION}

Total knee replacement (TKR) is performed in patients suffering from severe pain and functional limitations secondary to arthritis. The goal of a TKR is to reduce pain, restore function, correct mechanical mal alignment, ensure ligamentous balancing and restore the joint line [1]. In 2015 494,571 primary TKRs had been performed and reported to the Australian Orthopaedic Association National Joint Replacement Registry (AOANJRR) [2].
The most used primary total knee prostheses' in Australia are the Triathlon cruciate retaining (CR), Nexgen CR Flex and the Vanguard CR [2]. Despite the increasing number of TKRs performed each year, up to $20 \%$ of patients continue to report dissatisfaction [3-6]. Common reasons for dissatisfaction include residual pain, limited function, and crepitus [7-9]. Anterior knee pain affected up to 50\% of patients post-TKR, possibly due to patellofemoral mal-tracking, patellofemoral overstuffing, and patellar tilt $[7,8,10]$.
Quick Response Code:

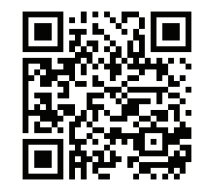

Address for correspondence: James Sires, College of Medicine and Public Health, Flinders University, Bedford Park, Australia

Received: June 29, 2020 Published: August 04, 2020

How to cite this article: Sires J, Lennon S, Inglis M, Nguyen A, Wilson C. Early Results of the Attune Knee System: A Minimum 2 Year Follow Up Observational Study. 2020 - 2(4) OAJBS. ID.000201. DOI: $10.38125 / O A J B S .000201$ 
The ATTUNE Knee System (DePuy Synthes, Warsaw) was designed in an effort to improve patient outcomes by providing more options for bearing and allowing easier kinematic balancing, as well as having a more anatomic patella and trochlear groove $[6,11,12]$. The ATTUNE Knee System has been used since 2011 and became widely available in 2013. While studies in the USA and Europe have shown good early outcomes there has been no data from Australian patients to date [11-13]. Therefore, the aim of this retrospective observational study was to investigate the early results of the ATTUNE TKR and ensure comparable results with other TKR systems used in Australia.

\section{MATERIAL AND METHODS}

An arthroplasty data system from a single institution was used to identify patients that had undergone TKR using the ATTUNE Knee System between $1^{\text {st }}$ September 2014 and 31 $1^{\text {st }}$ December 2015 allowing capture of the initial surgeries performed and a minimum follow-up of 2.0 years. Patients were selected to have an ATTUNE TKR if they fit the routine criteria in our department for a primary TKR surgery. The primary outcome was the need for revision surgery. Secondary outcomes included patient reported outcome, short-term postoperative range of motion (ROM) and surgical complications. Patients were included in the primary analysis if their primary diagnosis was osteoarthritis of the knee to allow for comparison with AOANJRR data. All patients were required to be $\geq 18$ years old. Exclusion criteria was revision surgery rather than primary TKR, as well as patients with primary diagnosis other than osteoarthritis. Ethics approval was gained from South Australian Local Health Network Human Research Ethics Committee. Patients were contacted either by mail or phone after their surgery and informed consent was gained from the patient to be included in the study.

A total of 162 TKRs, on 148 patients using the ATTUNE Knee System were included from our University teaching hospital. The surgeries were performed by one of 9 consultants or a supervised fellow using the medial para-patella approach. All but one TKR used a cruciate retaining technique as this was our surgeon's preference, with one TKR using a posterior stabilising technique. All cases in our learning curve with the ATTUNE implant were included. Postoperative all patients were referred to standard postoperative rehabilitation. ROM was collected at the 6 to 12 -week postoperative appointment and measured using a goniometer. Patient reported outcomes were measured using the Multi-Attribute Arthritis Prioritisation Tool (MAPT), which is a standardised and validated patient based score involving 11 multiple-choice questions asking the patient how their knee has impacted them over the previous three months $[14,15]$. The MAPT questionnaire was used as our routine patient reported functional assessment tool at the time this study started, and has been shown to be highly correlated with other questionnaires such as the Western Ontario and McMaster osteoarthritis index (WOMAC) and Oxford Knee, as well as demonstrating to be a good assessment of a patient's physical function and pain $[14,15]$. The MAPT assesses pain, limitations to daily activities, economic effects, recent deterioration and psychosocial health effects [16]. Individuals are sent a preoperative MAPT as part of routine care to aid in prioritisation of patients awaiting TKR, and since it is a validated functional score it is reasonable to compare preoperative and postoperative scores. The MAPT questionnaire produces a score ranging from 0 (least disease severity) to 100 (greatest disease severity) [14]. Previously, a MAPT score $\leq 20$ has been considered low priority for joint replacement surgery due to the patient having sufficient function [17] and therefore in this study a score of $\leq 20$ was considered a good outcome postoperatively. Conversely, a score $\geq 60$ has been previously considered high priority for joint replacement surgery [17] and therefore in this study if a patient had a score $\geq 60$ this was considered a poor outcome postoperatively.

Patients completed a preoperative MAPT questionnaire as part of their standard care. Additionally, patients were sent a MAPT to complete in either June or December 2017 to ensure a minimum time-to-follow up of 2.0 years. Non-responders were telephoned. Age, gender, date of TKR surgery and information regarding any surgical complication during or after surgery was collected from patients that received ATTUNE TKR at our University teaching hospital through electronic patient records.

To assess revision rates, TKRs involving the ATTUNE Knee System performed by the same surgeons at our private institution were included in the analysis. Total TKR with the ATTUNE Knee System performed at both institutions was 332. Figure 1 Highlights recruitment, inclusion and exclusion criteria. To identify ATTUNE TKRs that had received revision, two local databases were reviewed in December 2017. Additionally, an ad hoc report was requested and created from the AOANJRR to identify any other revisions that have occurred in Australia [18]. A total of 10 knees were excluded from the primary analysis as they received the ATTUNE Knee System for reasons other than osteoarthritis; rheumatoid arthritis (5), osteonecrosis (3), and other inflammatory arthritis (2).

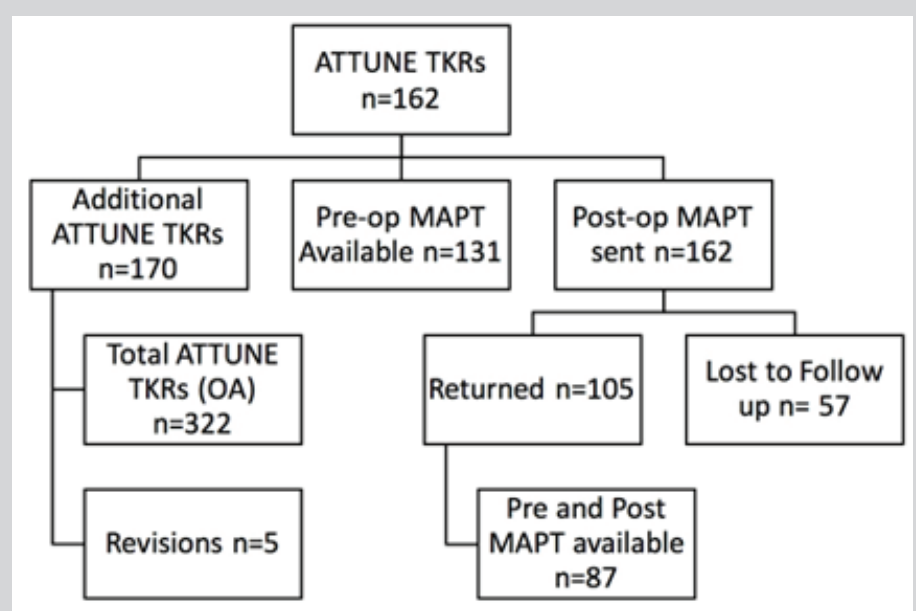

Figure 1: Patient flowchart showing inclusion and exclusion criteria for revision rate and MAPT questionnaires. 
Statistical analyses were performed using STATA 15.0. Z-score test statistic was used to test the difference in two population proportions of revision rates. MAPT score is reported as mean, median and standard deviation. Non-parametric Wilcoxon paired signed rank test was used to test the difference in median MAPT scores before and after TKR. Two-sample Wilcoxon rank-sum (Mann-Whitney) test for comparing postoperative MAPT scores between two age groups and MAPT scores between gender. A P value of $<0.05$ was considered significant.

\section{RESULTS}

The mean age of patients receiving TKR was 69.8 (46 to 86) years and $101(62.4 \%)$ were female. A total of 322 patients underwent TKR with the ATTUNE system between September 2014 and December 2015 for osteoarthritis of the knee as primary diagnosis. A total of five knees received revision surgery, revision rate of $1.6 \%$ with mean time to follow up of 2.6 years (2.0 to 3.2). Compared to ATTUNE TKR performed at other hospitals in Australia (0.7\%), there was no statistical difference in results $(p=0.090)$. Additionally, our revision rate was lower compared to other TKR nationwide $(2.1 \%)$, but there was no statistical difference in results $(p=0.508)$ (Table 1). Figure 2 illustrates local ATTUNE TKR cumulative revision rates alongside both other ATTUNE and other TKR in Australia [18]. One reason for revision included infection (2) $(0.6 \%)$, with both individuals receiving a change of insert. Additionally, one $(0.3 \%)$ knee underwent tibial and femoral component revision secondary to medial tibial plateau fracture. This was due to one of the pin sites on the tibial component being near the cortex of the bone, which in turn caused a stress riser creating a weak point for fracture, and subsequent loosening. Lastly one $(0.3 \%)$ knee underwent patella resurfacing secondary to patellofemoral pain, and one knee had a polyethylene exchange for instability $(0.3 \%)$.

Table 1: Revision Rates of primary ATTUNE, other ATTUNE and other total knee replacements.

\begin{tabular}{|c|c|c|c|}
\hline & Local Attune & Other Attune & Other Total Knee \\
\hline Primary TKRs & 322 & 7789 & 523975 \\
\hline Revisions & 5 & 56 & 10897 \\
\hline \% Revised & $1.60 \%$ & $0.70 \%$ & $2.10 \%$ \\
\hline$P$-Value & - & $0.090^{*}$ & $0.508^{* *}$ \\
\hline
\end{tabular}

${ }^{*}$ Z-scores comparing revision rates of ATTUNE to Other ATTUNE

${ }^{* *}$ Z-scores comparing revision rates of ATTUNE to Other Total Knee

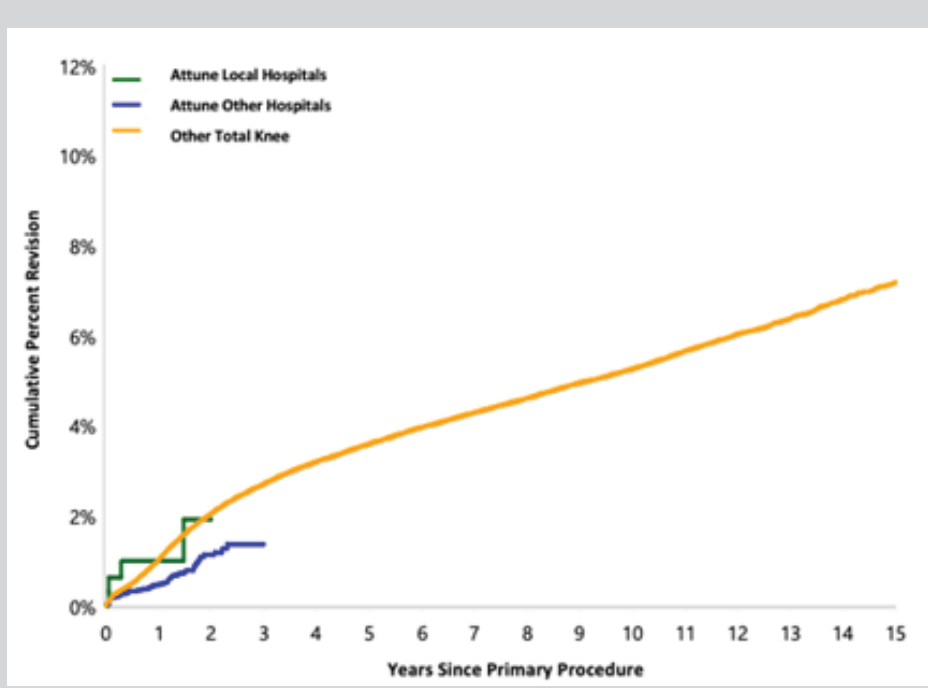

Figure 2: Cumulative Percent Revision of Primary Total Knee Replacement by Model (Primary Diagnosis OA) from the AOANJRR [20].

A total of 131 preoperative and 105 postoperative MAPT scores were collected, with 57 lost to follow-up (response rate 64.8\%). Mean follow-up for postoperative MAPT questionnaire was 2.4 years (2.0 to 2.8). The mean MAPT score prior to TKR, and at final follow-up was 62.8 (median 64.4, SD 27.4) and 10.2 (median 0.0, SD 21.2) respectively (Table 2). A total of 91 patients out of the 105 (86.7\%) who returned a postoperative MAPT had a MAPT score $\leq 20$. A total of $6(5.7 \%)$ patients had a MAPT score $\geq 60$.

When comparing patient gender, a total of 66 females had postoperative MAPT scores available, with a mean of 12.1 (median 0.0, SD 24.0) compared to male 6.8 (median 0.0, SD 15.1). There was no difference in the median postoperative MAPT scores between males and females ( $p=0.715)$. Additionally, mean post-op MAPT scores were lower in patients 65 years and over. A statistical difference in median postoperative MAPT scores between individuals was found, with <65 15.3 (median 2.6, SD 26.8) and individuals $\geq 657.7$ (median 0.0, SD 17.6) ( $p=0.033$ ).

A total of 87 knees had preoperative and postoperative MAPT scores available. In this group, mean preoperative and postoperative MAPT scores were 61.3 (median 63.4, SD 27.6) and 9.6 (median 0.0, SD 19.7) respectively, which was statistically different $(p<0.001)$. A total of 82 (94.3\%), out of 87 people had an improved MAPT score post-TKR. 
Table 2: Summary of MAPT Scores pre and postoperatively.

\begin{tabular}{|c|c|c|}
\hline & Preoperative MAPT ( $\boldsymbol{n}=\mathbf{1 3 1})$ & Postoperative MAPT ( $\boldsymbol{n}=\mathbf{1 0 5})$ \\
\hline Mean (Range) & $62.8(2.5-99.8)$ & $10.2(0.0$ to 97.9$)$ \\
\hline Median (IQR) & $64.4(38.6$ to 89.4$)$ & $0.0(0.0$ to 8.9$)$ \\
\hline MAPT $\leq 20$ & $6.90 \%$ & $86.70 \%$ \\
\hline MAPT $\geq 60$ & $56.50 \%$ & $5.70 \%$ \\
\hline Improved, $n(\%)$ & & $82(94.3 \%)^{*}$ \\
\hline
\end{tabular}

*82 of the 87 had improved postoperative MAPT score.

At 6-12 weeks postoperatively mean total ROM was $100.6^{\circ}$ (45 to 135 ) ( $n=153$ ). Mean flexion was $102.9^{\circ}(80$ to 130$)$, and mean extension was $3.3^{\circ}(-5$ to 30$)$.

A total of five out of the 162 TKR's required manipulations under anesthesia (3\%). A further [9] knees presented for investigation regarding knee pain, two patients were revised due to infection, one for fracture, one for patellofemoral pain and one for instability.

\section{DISCUSSION}

The ATTUNE Knee System has been used in Australia since 2013, and few studies are available looking at the outcomes of this new system. We looked at a combination of both surgical outcomes and a patient-based outcome to determine the early results of the ATTUNE TKR.

In our sample of 322 TKR's involving the ATTUNE Knee System for osteoarthritis a revision rate of $1.6 \%$ at mean time to follow up 2.6 years (2.0 to 3.2) was found. This was found to be higher compared to national ATTUNE TKR revision rates, but lower compared to all other TKR systems available in Australia. However, no statistically significant difference was found when comparing revision rates to either group. It is unknown whether no true difference exists between either cohort, this is because patient characteristics that may possibly influence implant survivorship were unavailable to be compared due to this study retrospective design. Additionally, as the ATTUNE Knee System is relatively new, and our series included all cases including ones in the learning curve, this may be one contributing factor to the increased revision rate in the local TKR cohort compared to ATTUNE TKR national rates.

An individual's osteoarthritis disease severity is measured by the Hip and Knee Multi-Attribute Prioritisation Tool. This tool aids surgeons in prioritising individuals requiring joint replacement surgery [14]. In this study, individuals were sent a preoperative MAPT as part of routine care, and therefore to assess and compare an individual's reported outcome with their TKR the MAPT questionnaire was used for a preoperative and postoperative comparison. Both construct validity and reliability have been validated previously in the MAPT questionnaire [14]. The MAPT has not been used to assess a patients' postoperative outcome previously, however for this study it was chosen because it is our institutions routine patient reported functional assessment tool at commencement of this study. One study that has used the MAPT postoperatively found a significant difference when comparing preoperative and postoperative MAPT scores and found 94.8\% of patients that had received a TKR had improved MAPT scores 6 months postoperatively [19]. In comparison, this study showed that at minimum two years $94.3 \%$ of patients that received an ATTUNE TKR had an improved MAPT score. In this study $86.7 \%$ of patients who returned a postoperative MAPT had a good outcome indicated by a MAPT score of $\leq 20$.

Other studies looking at patient satisfaction in individuals that had received an ATTUNE TKR found no statistical difference

in overall satisfaction compared to PFC Sigma [12]. Additionally, at two-year follow-up only $2.1 \%$ were found to be dissatisfied with their TKR, determined by a satisfaction score less than five on a visual analog scale. Ranawat et al. [12] discussed possible reasons for increased satisfaction of the ATTUNE TKR and included decreased anterior knee pain and crepitus compared to the PFC Sigma [12]. Furthermore, Indelli [13] used the Knee Society Score and Oxford Knee Score to assess clinical outcome of the ATTUNE TKR and found 98\% had good to excellent clinical outcomes, however this was not statistically different compared to the PFC sigma. Also, Martin [8] found patients implanted with a posterior stabilized ATTUNE TKR had much lower crepitus compared to the PFC Sigma at two years postoperatively ( $0.8 \%$ vs $9.4 \%$ ).

At 6-12 weeks postoperative patients had a mean total knee ROM of $100.6^{\circ}$. Guild and Labib [20] found a similar total ROM of $103.0^{\circ}$ in the NexGen LPS at 6 weeks. It should be noted that maximal postoperative ROM has not been recorded and it is expected to be higher at the two-year interval [21]. Studies that measured postoperative total knee ROM in patients who received the ATTUNE Knee System had a mean total knee ROM of $117.0^{\circ 12}, 123.0^{\circ 13}$ and $120.5^{\circ 22}$, similar to other TKR systems [13,22]. Additionally, due to this study's retrospective nature, preoperative total ROM and BMI were not collected and have previously been recognized to impact outcome [23]. Overall complication rate was relatively low, with an infection rate of $0.6 \%$ which is within the expected range.

\section{CONCLUSION}

In conclusion, our findings suggest the ATTUNE TKR has comparable revision rates to other TKRs currently available in Australia. Furthermore, patient reported outcome was high 2.4 years postoperatively, with a majority of patients having a good outcome. All patients in our learning curve were included and revision rates cross-referenced with the AOANJRR. This study was the first to use the MAPT questionnaire to assess patient reported outcomes post-TKR. Longer term-follow up of the ATTUNE Knee System is required to compare to other TKR systems available in Australia.

\section{REFERENCES}

1. Manen V, Mont NJ (2012) Management of primary knee osteoarthritis and indications for total knee arthroplasty for general practitioners. J Am Osteopath Assoc 112(11): 709-715.

2. (2016) Australian orthopaedic association national joint replacement registry (AOANJRR) 2016 annual report. Australia: Australian orthopaedic association.

3. Bourne RB, Chesworth BM, Davis AM, Mahomed NN, Charron K (2009) Patient satisfaction after total knee arthroplasty: who is satisfied and who is not? Clin Orthop Relat Res 468: 57-63.

4. Bourne RB, Chesworth B, Davis A, Mahomed N, Charron K (2010) Comparing patient outcomes after THA and TKA: is there a difference? Clin Orthop Relat Res 468(2): 542-546. 
5. Digennaro V, Zambianchi F, Marcovigi A (2014) Design and kinematics in total knee arthroplasty. Int Orthop 38(2): 227-233.

6. (2012) DePuy Synthes. Attune knee system.

7. Jacobs CA, Christensen CP (2014) Factors influencing patient satisfaction two to five years after primary total knee arthroplasty. The Journal of Arthroplasty 29: 1189-1191.

8. Martin JR, Jennings JM, Watters TS, Levy DL, McNabb DC, Dennis DA (2017) Femoral implant design modification decreases the incidence of patellar crepitus in total knee arthroplasty. The Journal of Arthroplasty 32: 1310-1313.

9. Young-Joon C, Ra HJ (1989) Patient satisfaction after total knee arthroplasty. Knee Surg Relat Res 28(1): 1-15.

10. Insall JN, Dorr LD, Scott RD (1989) Rationale of the knee society clinical rating system. Clin Orthop Relat Res 248: 13-14.

11. (2012) DePuy Synthes. Attune proving the promise.

12. Ranawat CS, White PB, West S, Ranawat AS (2017) Clinical and radiographic results of attune and pfc sigma knee designs at 2-Year Follow-Up: A prospective matched-pair analysis. The Journal of Arthroplasty 32(2): 431-436.

13. Indelli PF, Pipino G, Johnson P, Graceffa A, Marcucci M (2016) Posteriorstabilized total knee arthroplasty: a matched pair analysis of a classic and its evolutional design. Arthroplasty Today 2(4): 51-91.

14. Osborne R, Haynes K, Jones C, Chubb P, Robbins D, Graves S (2006) Orthopaedic waiting list project summary report. Victorian government department of human services, Melbourne, Australia.
15. Anouchi YS, McShane M, Kelly Jr F (1996) Range of motion in total knee replacement. Clin Orthop 331: 87-92.

16. (2012) DePuy Synthes. Attune proving the promise.

17. David VM, Bousounis G, Kapakoulakis T, Champion R, Masman K, et al. (2011) Correlation of MAPT scores with clinical and radiographic assessment of patients awaiting THR/TKR. ANZ J Surg 81(7-8): 543-546.

18. (2017) AOANJRR Ad hoc report. Report number 2131, Australia.

19. Nganga M, Bramwell D, Monaghan J, Doerr C, Mercer G, et al. (2018) Evaluation of the multi-attribute prioritisation tool for total joint replacement. Journal of Orthopaedics 15(1): 242-247.

20. Guild GN, Labib SA (2014) Clinical outcomes in high flexion total knee arthroplasty were not superior to standard posterior stabilized total knee arthroplasty. A multicenter, prospective, randomized study. The Journal of Arthroplasty 29(3): 530-534.

21. Kotani A, Yonekura, A, Bourne RB (2005) Factors influencing range of motion after contemporary total knee arthroplasty. J Arthroplasty 20(7): 850-856

22. Dwyer K, Jones RJ, Lesko J, Leopold J, Rodrigo D (2014) Attune knee system early performance: Minimum 2-year clinical results, DePuy Synthes.

23. Schroer WC, Stormont DM, Pietrzak WS (2014) Seven-year survivorship and functional outcomes of the high-flexion vanguard complete knee system. The Journal of Athroplasty 29: 61-65. 